\title{
Staphylococcus aureus Bacteremia: Comparison of Two Periods and a Predictive Model of Mortality
}

\author{
Lucieni de Oliveira Conterno, Sérgio Barsanti Wey \\ and Adauto Castelo
}

\author{
Division of Infectiuous Disease of Marília Medical \\ School, Division of Infectious Disease of Federal \\ University of São Paulo, SP, Brazil
}

\begin{abstract}
Staphylococcus aureus is an important pathogen causing bacteremia, primarily affecting hospitalized patients. We studied the epidemiology of $S$. aureus bacteremia, comparing two periods (early and mid 1990s) and developed a predictive model of mortality. A nested case-control was done. All 251 patients over 14 years old with positive blood cultures for $S$. aureus were selected. MRSA (methicillin resistant $S$. aureus) was isolated in $63 \%$ of the cases. When comparing the two periods MRSA community-acquired bacteremia increased from $4 \%$ to $16 \%(p=0.01)$. There was no significant difference in the mortality rate between the two periods $(39 \%$ and $33 \%, p=0.40)$. Intravascular catheters provoked $24 \%$ of the cases of bacteremia and were associated with the lowest rate of mortality. In a logistic regression analysis, three variables were associated with death: septic shock, source of bacteraemia and resistance to methicillin. The probability of dying among patients with MRSA and those with methicillin sensitive $S$. aureus bacteraemia ranged from $10 \%$ to $90 \%$ and from $4 \%$ to $76 \%$, respectively, depending on the source of the bacteraemia and the occurrence of septic shock. The MRSA found in Brazil may be a particularly virulent strain. Key Words: Staphylococcus aureus, bacteremia, mortality.
\end{abstract}

In recent years, methicillin resistant Staphylococcus aureus (MRSA) has emerged as an important pathogen, primarily affecting hospitalized patients, and has become one of the leading causes of nosocomial bacteremia [1-7].

Several studies have indicated no difference in virulence when clinical outcomes in patients with infection due to MRSA were compared with those with methicillin-sensitive $S$. aureus (MSSA) infection. However, other studies indicate that methicillin resistance is a risk factor for a poor outcome in patients with $S$. aureus bacteremia [8-12]

Received on 15 May 2002; revised 12 December 2002. Address for correspondence: Dr. Lucieni de Oliveira Conterno Rua José Camarinha 467, Bairro Maria Isabel. Zip Code: 17516 220 Marília, São Paulo, Brasil. E-mail: lucieni@famema.br This study was supported by INCLEN- International Clinical Epidemiology network.

The Brazilian Journal of Infectious Diseases 2002;6(6):288-297 (C) 2002 by The Brazilian Journal of Infectious Diseases and Contexto Publishing. All rights reserved. $1413-8670$
We compared $S$. aureus bacteremia, during two study periods: 1991-1992 and 1995-1996, and developed a predictive model for mortality.

\section{Material and Methods}

Design. The cohort of all adult patients at São Paulo hospital with $S$. aureus bacteremia, during 1990-1991 and 1995-1996 was studied. These two periods were chosen to evaluate the trends of methicillin resistance rate in the hospital and to determine if there were changes in the treatment and evolution of $S$. aureus bacteremia after a more strict antibiotic control policy was implemented at this hospital in 1993. A nested case-control study was done to identify risk factors for mortality. Patients with $S$. aureus bacteremia who died were considered the study cases and the patients with $S$. aureus bacteremia who survived were chosen as controls.

Setting. The study was carried out in the São Paulo Hospital, Brazil, which has a high prevalence rate of 
MRSA. It is a 600-bed, university, general public hospital.


culture positive for $S$. aureus were selected. A review of medical records was made to characterize the clinical picture, signs of infection in other sites within 48 hours of the blood culture, drugs used and clinical progress up to the 14th day of bacteraemia. The 14th day after bacteremia was chosen because most deaths related to bacteremia occurred early, normally not later than the second week.

Bacteremia was defined by at least one blood culture positive for $S$. aureus. Patients were grouped, according to clinical manifestation as: (1) Sepsis, (2) Severe sepsis or (3) Septic shock [2]. The clinical manifestation was assessed at the time when the first antibiotic treatment was defined. Nosocomial bacteremia was defined as bacteremia occurring 48 hours or more after hospital admission; bacteremia in patients on hemodialysis or those receiving outpatient intravenous therapy were also defined as nosocomial [13].

Sources of bacteraemia were defined if there was either clinical or bacteriological evidence of infection at another site within 48 hours of the positive blood culture.

The initial antibiotic therapy was considered to be adequate if at least one antibiotic to which the bacterium was sensitive, in vitro, had been used within the first 48 hours after blood culture material collection; corrected whenever the antibiotic used within the first 48 hours had to be replaced by another antibiotic because the microorganism was found to be resistant in vitro; and inadequate when the agent was resistant, in vitro to the antibiotic used within the first 48 hours, and no other antibiotic was used in its place.

Deaths were attributed to bacteremia if they occurred within 14 days of the first positive blood culture and without any other obvious cause.

The Pearson chi-squared test or Fisher's exact test was used to compare proportions. The periods of study were analyzed to determine how they affected mortality, because there were differences in both the proportions of patients with adequate treatment and the principal source of bacteremia between the two periods. Variables showing significant associations in the univariate analysis were further analyzed by logistic regression. The logistic regression model evaluated interactions among treatment, infection source, septic shock and methicillin resistance. The predictive model of mortality was developed using coefficients calculated for each of the variables selected by the logistic regression analysis.

\section{Results}

Over 100 patients with $S$. aureus bacteremia were identified during each of the two periods (Table1). Most patients were men 60-years old or under, and most bacteremias were hospital acquired. More than $80 \%$ of the patients had one underlying disease.

In a comparison between the two periods, the patients did not differ significantly in terms of age, sex, presence of underlying disease, occurrence of septic shock or frequency of MRSA isolated by blood culture. In the first period, $66 \%$ of the strains were MRSA, which was similar to that found in the second period $(60 \%, p=0.31)$. The catheter was considered to be the source of bacteremia in $24 \%$ of the patients during the two periods, analyzed together.

The proportion of bacteremias with undetermined source decreased from the earlier to the later period by $24 \%$ (95\% CI: $12 \%, 35 \%$; $<<0.01)$. The proportion of bacteremias with a respiratory tract source increased by $13 \%$ (95\% CI: $22 \%, 30 \%$; p <0.01), and bacteremias with other sources increased by $14 \%$ (95\% CI: $-24 \%,-4 \% ; \mathrm{p}<0.01$ ). There was an increase in the proportion of community-acquired bacteremia from the first to the second period(from $14 \%$ to $28 \%$; $\mathrm{p}=0.01$ ). This increase was largely due to community acquired MRSA bacteremia, which had a frequency of $4 \%$ in the first period and $16 \%$ in the second $(\mathrm{p}<0.01)$.

A significantly higher proportion of patients received adequate or corrected treatment in the second than in the first period $(69 \%$ and $83 \%$; $p<0.01)$. There was an improvement of the treatment for bacteremia by 
Table 1. Characteristics of patients with Staphylococcus aureus bacteremia

\begin{tabular}{|c|c|c|c|c|c|}
\hline & $\begin{array}{l}\text { 1991-1992 } \\
\text { No. }(\%)\end{array}$ & $\begin{array}{l}\text { 1995-1996 } \\
\text { No. }(\%)\end{array}$ & $\mathbf{P}$ & $\begin{array}{c}\text { Difference } \\
\%\end{array}$ & CI $95 \%$ \\
\hline Number of cases & 136 & 115 & & & \\
\hline \multicolumn{6}{|l|}{ Age } \\
\hline$<60$ years & $71 \%$ & $75 \%$ & 0.38 & $4 \%$ & $15 \%, 7 \%$ \\
\hline \multicolumn{6}{|l|}{ Sex } \\
\hline Male & $62 \%$ & $61 \%$ & 0.88 & $1 \%$ & $-11 \%, 13 \%$ \\
\hline Community acquired & $14 \%$ & $28 \%$ & 0.01 & $14 \%$ & $-24 \%,-4 \%$ \\
\hline $\begin{array}{l}\text { Underlying disease } \\
\text { present }\end{array}$ & $85 \%$ & $86 \%$ & 0.85 & $1 \%$ & $-10 \%, 8 \%$ \\
\hline \multicolumn{6}{|l|}{ Source of bacteremia } \\
\hline Catheter & $26 \%$ & $23 \%$ & & $3 \%$ & $-7 \%, 14 \%$ \\
\hline Respiratory tract & $12 \%$ & $25 \%$ & & $13 \%$ & $-22 \%,-3 \%$ \\
\hline Others & $15 \%$ & $29 \%$ & & $14 \%$ & $24 \%, 4 \%$ \\
\hline Undetermined & $47 \%$ & $23 \%$ & 0.001 & $24 \%$ & $12 \%, 35 \%$ \\
\hline MRSA & $66 \%$ & $60 \%$ & 0.31 & $6 \%$ & $-6 \%, 18 \%$ \\
\hline \multicolumn{6}{|l|}{ Clinical picture } \\
\hline Sepsis & $60 \%$ & $50 \%$ & & $10 \%$ & $-2 \%, 22 \%$ \\
\hline Severe sepsis & $17 \%$ & $19 \%$ & & $2 \%$ & $-11 \%, 8 \%$ \\
\hline Septic shock & $22 \%$ & $30 \%$ & 0.24 & $8 \%$ & $-19 \%, 26 \%$ \\
\hline \multicolumn{6}{|l|}{ Treatment } \\
\hline Adequate/ corrected & $69 \%$ & $83 \%$ & 0.01 & $14 \%$ & $-24 \%, 3 \%$ \\
\hline Death & $39 \%$ & $33 \%$ & 0.40 & $6 \%$ & $-6 \%, 17 \%$ \\
\hline
\end{tabular}

MRSA: methicillin resistant $S$. aureus.

MRSA, with $58 \%$ of the patients being treated appropriately in 1991-1992 and 77\% in 1995-1996, $(\mathrm{p}=0.008)$. There was nosignificant differencein themortality rate between the time periods ( $39 \%$ and $33 \%$; $\mathrm{p}=0.40$ ).

Table 2 shows the statistical association among several clinical variables and death in the two periods. There were no significant differences for any of the variables across time. Therefore the analysis was done considering all patients of each of the two periods together.

The univariate analysis showed that age above sixty years, hospital acquired bacteremia, methicillin resistance, respiratory and undetermined sources of bacteremia, occurrence of septic shock and inadequate treatment were significantly associated with mortality (Table 3). Mortality remained significantly associated with bacteremia by MRSA, source of bacteremia and occurrence of shock septic, when the patients were grouped based on inadequate versus adequate treatment was done (Table 4).

Logistic regression analysis with stepwise backward elimination of variables was applied. At first, the terms of interaction: treatment $\mathrm{X}$ methicillin resistance, treatment $X$ septic shock, treatment $X$ source of bacteraemia and methicillin resistance $X$ source were included in the logistic model. The likelihood-ratio test, comparing the model with and without interaction terms, showed no significant difference $(\mathrm{p}=0.23)$.

The logistic regression analysis selected three variables that were significantly associated with death: septic shock, methicillin resistance, and source of bacteremia (Table 5). 
Table 2. Association between clinical variables and death by day 14 in cases of Staphylococcus aureus bacteremia during each of two periods of study (1991-1992 and 1995-1996)

\begin{tabular}{|c|c|c|c|c|c|c|}
\hline & \multicolumn{3}{|c|}{ First period } & \multicolumn{3}{|c|}{ Second period } \\
\hline & Survival & Death & $\mathbf{P}$ & Survival & Death & $\mathbf{P}$ \\
\hline$\geq 60$ years & 19 & 22 & & 15 & 14 & \\
\hline$<60$ years & 64 & 31 & 0.02 & 61 & 25 & 0.15 \\
\hline Hospital acquired & 67 & 50 & & 59 & 24 & \\
\hline Community acquired & 16 & 3 & 0.02 & 17 & 15 & 0.06 \\
\hline \multicolumn{7}{|l|}{ Underlying disease } \\
\hline Present & 70 & 46 & & 64 & 35 & \\
\hline Absent & 13 & 7 & 0.80 & 12 & 4 & 1.00 \\
\hline MRSA & 46 & 44 & & 40 & 29 & \\
\hline MSSA & 37 & 9 & 0.01 & 36 & 10 & 0.03 \\
\hline \multicolumn{7}{|l|}{ Source of bacteremia } \\
\hline Catheter & 31 & 4 & & 20 & 6 & \\
\hline Respiratory tract & 6 & 11 & & 16 & 13 & \\
\hline Others & 14 & 6 & & 26 & 7 & \\
\hline Undetermined & 32 & 32 & $<0.001$ & 14 & 13 & 0.05 \\
\hline With Septic shock & 8 & 22 & & 12 & 23 & \\
\hline Without Septic shock & 75 & 31 & $<0.001$ & 64 & 16 & $<0.001$ \\
\hline Inadequate treatment & 18 & 24 & & 9 & 11 & \\
\hline Adequate treatment & 65 & 29 & 0.004 & 76 & 39 & 0.02 \\
\hline
\end{tabular}

MRSA: methicillin resistant S. aureus; MSSA: methicillin sensitive S. aureus.

The predictive model of mortality considering the occurrence of septic shock and source of bacteremia (Table 6) shows that the probability of dying ranged from $10 \%$ to $90 \%$, among patients with MRSA bacteremia; and among patients with MSSA bacteremia, the probability of dying varied from $4 \%$ to $76 \%$.

\section{Discussion}

S. aureus is one of the main agents of hospital acquired bloodstream infection [5,14]. In our study most of the $S$. aureus bacteremias were hospital acquired, and accounted for $86 \%$ and $72 \%$ of the cases in each of the two periods. The $S$. aureus strains isolated at São Paulo Hospital, had a high frequency of resistance to antibiotics, but these rates were similar in the two study periods ( $66 \%$ and $60 \%, \mathrm{p}=0.31$ ).

Although MRSA is often thought of a nosocomial agent, there are increasing reports of MRSA community acquired infections [15-17]. We observed that $4 \%$ of the MRSA bacteremias were community acquired, in the first period, while in the second period the rate increased to $16 \%(\mathrm{p}<0.01)$. It is known that patients who are colonized by MRSA in the hospital can sustain the infection for 6 to 12 months after discharge. They can serve as a source in the community and be readmitted to the same or another hospital [18]. Some of the MRSA cases that were considered community acquired could in fact have been hospital acquired. Nevertheless, it is important to be aware that an increasingly number of patients already infected with 
Table 3. Association between selected clinical factors and risk of death following Staphylococcus aureus bacteremia, considering all patients $(n=251)$

\begin{tabular}{|c|c|c|c|c|c|}
\hline & Survival & Death & $\mathbf{P}$ & OR & $95 \% \mathrm{CI}$ \\
\hline Number & 159 & 92 & & & \\
\hline$<60$ years & 125 & 56 & & & \\
\hline$\geq 60$ years & 34 & 36 & 0.003 & 0.46 & $0.25-0.84$ \\
\hline Hospital-acquired bacteremia & 126 & 74 & & & \\
\hline Community-acquired bacteremia & 33 & 18 & 0.82 & 1.0 & $0.57-2.0$ \\
\hline \multicolumn{6}{|l|}{ Underlying disease } \\
\hline Absent & 25 & 11 & & & \\
\hline Present & 134 & 81 & 0.41 & 1.4 & $0.65-2.9$ \\
\hline MRSA & 86 & 73 & & & \\
\hline MSSA & 73 & 19 & $<0.001$ & 3.3 & $1.8-5.9$ \\
\hline \multicolumn{6}{|l|}{ Source of bacteremia } \\
\hline Catheter & 51 & 10 & & 0.6 & $0.2-1.6$ \\
\hline Respiratory tract & 22 & 24 & & 3.6 & $1.3-8.6$ \\
\hline Undetermined & 46 & 45 & & 3.0 & $1.3-6.8$ \\
\hline Others & 40 & 13 & $<0.001$ & 1.0 & - \\
\hline With septic shock & 20 & 45 & & & \\
\hline Without septic shock & 139 & 47 & $<0.001$ & 6.65 & $3.6-12.3$ \\
\hline Inadequate treatment & 27 & 35 & & & \\
\hline Adequate treatment & 132 & 57 & $<0.001$ & 3.0 & $1.6-5.41$ \\
\hline
\end{tabular}

MRSA: methicillin resistant $S$. aureus. MSSA: methicillin resistant $S$. aureus. OR: odds ratio; $95 \%$ CI: confidence interval; Others: tegument; surgery infection.

MRSA are being admitted into hospitals for treatment. The intravascular catheter was the source of bacteraemia in $26 \%$ and $23 \%$ of patients in the two periods of this study and $24 \%$ overall. Bacteremia is one of the known complications of infection provoked by intravascular catheters. These data are similar to the situation described by Pittet et al. [19], who analyzed 1090 episodes of primary bacteremia and found 212 cases (19\%) associated with vascular catheters, and in $74 \%$ of these, the agent was Staphylococcus sp.

In our study, , the respiratory tract was considered to be the source of the bacteremia in $12 \%$ and $25 \%$ of the cases, in the two periods. Similarly, Taylor et al.[20] identified S. aureus in $27 \%$ of the nosocomial pneumonia cases that presented a bacteraemia. The patients at high risk of developing nosocomial pneumonia generally have other risk factors for acquiring infections [21,22].
There was an evident improvement, from the first to the second period, in the adequate use of antibiotics in MRSA bacteremia. This might be due to (1) improved physicians knowledge concerning the resistance pattern of the most frequently isolated bacteria in the hospital; (2) a more strict antibiotic control policy adopted by Hospital Sao Paulo after 1993; in that year an infectious disease specialist was responsible for the surveillance of all blood cultures or (3) a decrease in the bacteremia rate from undetermined sources. Thus, the agents involved could be better predicted and an improvement in the initially empirical antibiotic treatment was made possible. Leibovice et al. [23] found inadequate antibiotic treatment to be more frequent among cases with undetermined source bacteremia than those with known source $(49 \%$ and $35 \%$ respectively; $\mathrm{p}<0.001)$. 
Table 4. Association between selected clinical factors and death after Staphylococcus aureus bacteremia, stratified by treatment situation

\begin{tabular}{|c|c|c|c|}
\hline & Survival & Death & $\mathbf{P}$ \\
\hline \multicolumn{4}{|l|}{ Adequate treatment } \\
\hline First period (94) & $69 \%$ & $31 \%$ & \\
\hline Second period $(95)$ & $70 \%$ & $30 \%$ & 0.87 \\
\hline MRSA (104) & $62 \%$ & $38 \%$ & \\
\hline $\operatorname{MSSA}(85)$ & $79 \%$ & $21 \%$ & 0.01 \\
\hline Without septic shock (143) & $79 \%$ & $21 \%$ & \\
\hline With septic shock (46) & $41 \%$ & $59 \%$ & $<0.001$ \\
\hline \multicolumn{4}{|l|}{ Source of bacteraemia } \\
\hline Catheter (53) & $85 \%$ & $15 \%$ & \\
\hline Respiratory tract (38) & $55 \%$ & $45 \%$ & \\
\hline Undetermined (54) & $57 \%$ & $43 \%$ & \\
\hline Others (44) & $79 \%$ & $21 \%$ & 0.001 \\
\hline \multicolumn{4}{|l|}{ Inadequate treatment } \\
\hline First period (42) & $57 \%$ & $43 \%$ & \\
\hline Second period (20) & $55 \%$ & $45 \%$ & $<0.001$ \\
\hline $\operatorname{MRSA}(55)$ & $38 \%$ & $62 \%$ & \\
\hline $\operatorname{MSSA}(7)$ & $86 \%$ & $14 \%$ & 0.01 \\
\hline Without septic shock (43) & $60 \%$ & $40 \%$ & \\
\hline With septic shock (19) & $5 \%$ & $95 \%$ & $<0.001$ \\
\hline \multicolumn{4}{|l|}{ Source of bacteremia } \\
\hline Catheter (8) & $75 \%$ & $25 \%$ & \\
\hline Respiratory tract (8) & $12 \%$ & $88 \%$ & \\
\hline Undetermined (37) & $40 \%$ & $60 \%$ & \\
\hline Others (9) & $55 \%$ & $45 \%$ & 0.05 \\
\hline
\end{tabular}

MRSA: methicillin resistant $S$. aureus. MSSA: methicillin resistant S. aureus. Others: tegument; surgery infection.

Mortality within 14 days was chosen as the cutoff, because several authors have suggested that death due to bacteremia occurs early, rarely after the second week. The observed mortality was $39 \%$ and $33 \%$ in each period ( $\mathrm{p}=0.43$ ), and was associated with source of bacteremia, inadequate treatment, and occurrence of septic shock and methicillin resistance. The source of bacteremia, occurrence of septic shock and methicillin resistance was associated with death, even when adjusted by the treatment received.

The overall mortality rate was $37 \%$, similar to that found by Brum-Buisson, Doyon and Carlet [24], in their study in 24 French hospitals, where the bacteremia mortality rate ranged from $25 \%$ to $54 \%$, depending on the clinical presentation.

Mortality due to S. aureus bacteremia provoked by catheter contamination is reported to be about $15 \%$ [25]. We found a similar percentage (16\%) of patients who died from contamination by the vascular catheter. It is believed that bacteremias caused by catheters lead to lower rates of mortality and complications, since they are more easily diagnosed, and more promptly treated. S. aureus is therefore more quickly eradicated as the source of infection is removable [26].

Nosocomial pneumonia is associated with an increased death risk, ranging from $20 \%$ to $84 \%$ [27- 
Table 5. Clinical variables found by logistic regression analysis to have a significant association with risk of death by day 14 following Staphylococcus aureus bacteremia, considering all 251 patients

\begin{tabular}{lclllc}
\hline Variable & $\begin{array}{c}\text { Regression } \\
\text { coefficient }\end{array}$ & SE & P & OR & 95\% CI \\
\hline $\begin{array}{l}\text { Source of bacteremia } \\
\quad \text { Catheter }\end{array}$ & -1.09 & 0.32 & $<0.001$ & 0.33 & $0.17-0.63$ \\
$\quad$ Respiratory tract & 0.73 & 0.29 & 0.01 & 2.07 & $1.27-3.37$ \\
$\quad$ Undetermined & 0.99 & 0.25 & $<0.001$ & 2.68 & $1.63-4.41$ \\
$\quad$ Others & - & - & - & 1.0 & - \\
Septic shock & 1.15 & 0.19 & $<0.001$ & 3.15 & $2.16-4.58$ \\
No shock & - & - & - & 1.0 & - \\
Methicillin resistance & 0.58 & 0.17 & 0.002 & 1.80 & $1.27-2.54$ \\
Methicillin susceptibility & - & - & - & 1.0 & - \\
1st Period of study & 0.63 & 0.19 & 0.43 & & - \\
2nd period of study & - & - & - & 1.0 & \\
Constant & -0.43 & & & & \\
\hline
\end{tabular}

SE: standard error; OR: odds ratio; 95\% CI: confidence interval.

Table 6. Predictive model of mortality among patients with Staphylococcus aureus bacteremia, considering the coefficient of logistic regression analysis

Methicillin sensitive $S$. aureus

Clinical picture

\begin{tabular}{lcc} 
& No shock & Septic shock \\
\cline { 2 - 3 } Source of bacteremia & & \\
Catheter & $4 \%$ & $28 \%$ \\
Respiratory tract & $20 \%$ & $71 \%$ \\
Unknown & $24 \%$ & $76 \%$ \\
Others & $8 \%$ & $62 \%$ \\
\hline & \multicolumn{2}{c}{ Methicillin resistant S. aureus } \\
& \multicolumn{2}{c}{ Clinical picture } \\
\cline { 2 - 3 } & No shock & Septic shock \\
\hline Source of bacteremia & \multicolumn{2}{c}{$5 \%$} \\
Catheter & $10 \%$ & $88 \%$ \\
Respiratory tract & $42 \%$ & $90 \%$ \\
Unknown & $48 \%$ & $82 \%$ \\
Others & $21 \%$ & \\
\hline
\end{tabular}


30]. In our study, $52 \%$ of the patients with $S$. aureus bacteremia, involving the respiratory tract, died. RelloTorres et al. [22], also reported a higher mortality rate among patients with MRSA pneumonia, compared with pneumonia caused by sensitive strains ( $R R=20.72$; $\mathrm{CI}=95 \%: 2.78-154.3)$.

Septic shock is considered to be an important death risk factor among patients with bacteremia [24,29]. The mortality observed by Lundberg et al. [31] among patients who developed septic shock ranged from $39 \%$ to $70 \%$. They suggested that delay in the treatment of shock has a significant impact on mortality due to sepsis. In our study, 22/30 of patients with septic shock died. Most patients, even with septic shock, were in hospital wards, and just 9.6\% were in Intensive Care Units. Patients in hospital wards may have been treated later and less aggressively, which could explain the observed outcome.

Today there are still doubts about the contribution of methicillin resistance to mortality due toinfections caused by $S$. aureus. In our study, patients with MRSA bacteremia had a higher probability of dying compared with patients with MSSA bacteremia (46\% and 22\%, respectively, $\mathrm{OR}=3.3, \mathrm{CI}=95 \%$ : $1.8-5.9$ ). Even among those who received adequate treatment, MRSA bacteremia resulted in a higher mortality rate than MSSA bacteremia ( $38 \%$ and $21 \%$ respectively, $\mathrm{p}<0.01$ ).

The higher mortality rate among patients with MRSA bacteremia was similar to the results of others author [32]. Romero-Vivas et al.,[11] also observed, in a logistic regression analysis, higher mortality rates among patients with bacteremia caused by methicillin resistant strains, even when considering only the patients who received appropriate treatment $(\mathrm{OR}=3.0 \mathrm{CI}=95 \%$ : 1.44-6.25).

Blot et al. [33] investigated outcomes in critically ill patients with $S$. aureus bacteremia. Logistic regression analysis showed that methicillin resistance and an unfavorable APACHE II score were independent risk factors for death $(\mathrm{p}<0.0001)$.

Selvey et al. [12] compared nosocomial bacteremia caused by MRSA and MSSA. They found a higher mortality rate among MRSA bacteremia patients (13.8 and 8.2, $\mathrm{p}<0.05)$.
The risk factors for acquiring MRSA may also influence the evolution of the bacteremia. We were unable to control many confounding factors observed among hospitalized patients. The higher mortality rate observed among the cases of MRSA bacteremia may reflect misclassification of cases, different populations of patients, or types of treatment provided to the patients. On the other hand, the differences could be due to specific microbiological characteristics of the strains isolated in Brazil.

The current geographical variation among the most frequently isolated clones in different locations can partially explain the differences observed in the MRSA infection history. In Brazil, the SP genotype profile, determined by pulsed field gel electrophoresis, is the most common, and it was the most prevalent isolated MRSA clone in our study (86\%) [34]. This clone may have specific virulence characteristics that are different from those of other clones.

Higher mortality rates reported for infections with resistant strains have been described in Europe, mainly in the Iberian Peninsula. It has been recently found that the profile found in Brazil is similar to that of isolated strains in Portugal and Spain. This fact reinforces the possibility of differential virulence among the several MRSA strains, and perhaps, that the clone, peculiar to Brazil, Spain and Portugal is more virulent [29,35-37]

Predictive model of mortality. The estimates of death probabilities calculated for each subgroup of patients. suggest a great impact of septic shock on the course of the $S$. aureus bacteremia. The probability of dying is extremely high when shock occurs, except when the catheter is the source. Comparing patients with the same source of infection, and the same clinical presentation, the MRSA bacteremia patients have a higher probability of dying than those with MSSA bacteraemia (10\% vs. $4 \%$ and $90 \%$ vs $76 \%$, respectively).

\section{Conclusion}

An increase in MRSA community- acquired bacteraemia was observed when the two periods 
(1991-1992 and 1995-1996) were compared. One can estimate the probability of each patient dying by considering the variables simultaneously, these being source of infection, occurrence of septic shock, and susceptibility to methicillin.

\section{Acknowledgements}

We are very grateful to Dr. Stephen Walters for his suggestions on the paper.

\section{References}

1. Banerjee S.N., Emori T.G., Culver D.H., et al. Secular trends in nosocomial primary bloodstream infections in the United States, 1980-1989. National Nosocomial Infections Surveillance System. Amer J Med 1991;91(3B): S86-9.

2. Bone R.C.,Balk, R.A., Cerra F.B., et al. Definitions for sepsis and organ failure and guidelines for the use of innovative therapies in sepsis. Chest 1992; 101:1644-55.

3. Jarvis W.R., Martone W.J. Predominant pathogens in hospital infections. J. Antimicrob. Chemother 1992;29(suppl. A):19-24.

4. McDonald M., The epidemiology of methicillin-resistant Staphylococcus aureus: surgical relevance 20 years on. Aust N Z J Surg 1997;67:682- 5.

5. Vincent J.L., Bihardi D.J., Suter P.M., et al. The prevalence of nosocomial infection in intensive care units in Europe: results of the European Prevalence of Infection in Intensive Care (EPIC) Study. J Amer Med Ass 1995; 74:639-44.

6. Weistein M.P.,Towns M.L. Quartey S.M., et al. The clinical significance of positive blood cultures in the 1990s: a prospective comprehensive evaluation of the microbiology, epidemiology, and outcome of bacteremia and fungemia in adults. Clin Infect Dis 1997;24:584-602.

7. Cunney R.J., McNamara E.B., Ansari N., SMYTH E.G. Community and hospital acquired Staphylococcus aureus septicaemia: 115 cases from a Dublin teaching hospital. J Infect Dis 1996;33:11-3.

8. IwaharaT., Ichiyama S., Nada T., et al. Clinical and epidemiologic investigations of nosocomial pulmonary infections caused by methicillin-resistant Staphylococcus aureus. Chest 1994;105:826-31.

9. Marty L., Flahault A., Suarez B., et al. Resistance to methicillin and virulence of Staphylococcus aureus strains in bacteremic cancer patients. Intens Care Med 1993; 19:285-9.
10. Romero-VivasJ., Rubio M., FernandeC., Picazo J.J. Mortality associated with nosocomial bacteremia due to methicillin-resistant Staphylococcus aureus. Clin Infect Dis 1995;21:1417-23.

11. Selvey L.A., Whiby M., Johnson B. Nosocomial Methicillin resistant Staphylococcus aureus bacteremia: is it worse than nosocomial methicillinsensitive Staphylococcus aureus bacteremia? Infect Control Hosp Epidem 2000;21:645-8.

12. Soriano A., Martinez J.Á., Mensa J., et al. Pathogenic significance of methicillin resistance for patients with Staphylococcus aureus bacteremia. Clin Infect Dis 2000;30:368-73.

13. Gardner J.S., Jarvis W.R., Emori G.T, et al. CDC definitions for nosocomial infections. Amer J Infect Control 1988; $16: 128-40$.

14. Jones R. Impact of changing pathogens and antimicrobial susceptibility patterns in the treatment of serious infections in hospitalized patients. Amer J Med 1996; 100(6A):S3-S12.

15. Embil J., Ramotar K., Romance L., et al. Methicillinresistant Staphylococcus aureus in tertiary care institutions on the Canadian prairies 1990-1992. Infect Control Hosp Epidem1994;15:646-51.

16. Laytor M.C., Hierholzer W.J., Patterson J.E.. The evolving epidemiology of methicillin-resistant Staphylococcus aureus at a university hospital. Infect Control Hosp Epidem1995;6:12-7.

17. Steinberg J.P., Clarck C.C., Hackman B.O. Nosocomial and community-acquired Staphylococcus aureus bacteremias from 1980 to 1993: impact of intravascular devices and methicillin resistance. Clin Infect Dis 1996;23:253-9.

18. Boyce J.M. Are the epidemiology and microbiology of methicillin-resistant Staphylococcus aureus changing? J Amer Med Ass 1998;279:623-4.

19. Pittet D., Woolson R.F., Wenzel R.P. Microbiological factors influencing the outcome of nosocomial bloodstream infections: a 6-year validated, populationbased model. Clin Infect Dis 1997;24:1068-78.

20. Taylor G.D., Buchanan-Chell M., Kirkland T., et al. Bacteremic nosocomial pneumonia: a 7-year experience in one institution. Chest 1995;108:786-8.

21. Leibovici L., Druncker M., Konisberger H., et al. Septic shock in bacteremic patients: risk factors, features and prognosis. Scand J Infect Dis 1997;29:71-5.

22. Rello J., Torres A., Ricart, M., et al. Ventilator-associated pneumonia by Staphylococcus aureus: comparison of methicillin-resistant and methicillin- sensitive episodes. Amer J Resp Crit. Care Med 1994;150:1545-9.

23. Brun-Bruisson C., Doyon F., Carlet J. Bacteremia and severe sepsis in adults: a multicenter prospective survey in ICUs and wards of 24 hospitals. Amer J Respir Crit Care Med 1996;154:617-24. 
24. Jeribgan J.A., Farr B.M. Short-course therapy of catheterrelated Staphylococcus aureus bacteremia: a metaanalysis. Ann Intern Med 1993;119: 304-11.

25. Malanoski G.J., Samore M.H., Pefanis A., Karchmer A.W. Staphylococcus aureus catheter- associated bacteremia. Arch Intern Med 1995; 155:1161-6.

26. Fagon J.Y., Maillet J.M. , Novara A. Hospital-acquired pneumonia: methicillin resistance and intensive care unit admission. Amer J Med 1998; 104(5A):S17-23.

27. Hershow R.C., Khayr W.F., Smith N.L. A comparison of clinical virulence of nosocomially acquired methicillinresistant and methicillin-sensitive Staphylococcus aureus infections in a university hospital. Infect Control Hosp Epidem 1992;13:587-93.

28. Jonhston B.L. Methicillin-resistant Staphylococcus aureus as a cause of community- acquired pneumonia: a critical review. Semin Resp Infect 1994;9:199-206.

29. Pittet D., Tarara D., Wenzel R.P. Nosocomial bloodstream infection in critically ill patients. J Amer Med Ass 1994; 71:1598-1601.

30. Lundberg J.S., Perl T.M., Winblin T., et al. Septic shock: an analysis of outcomes for patients with onset on hospital wards versus intensive care units. Crit Care Med 1998;6:1020-4.

31. Blot S.B., Vandewoude K., Colardyn F. Staphylococcus aureus infections. New Engl J Med 1998;339:2024-5.

32. Musser J.M., Kapur V. Clonal analysis of methicillinresistant Staphylococcus aureus strains from intercontinental sources: association of the mec gene with divergent phylogenetic lineages implies dissemination by horizontal transfer and recombination. J Clin Microbiol 1992;30:2058-63.

33. Conterno L.O, Wey S.B., Pignatary A.C., et al. Genotyping of MRSA isolated in blood culture (in press).

34. Oliveira D., Santos-Sanches I., Mato R. Virtually all methicillin-resistant Staphylococcus aureus (MRSA) infections in the largest Portuguese teaching hospital are caused by two internationally spread multiresistant strains: the "Iberian" and the "Brazilian" clones of MRSA. Clin Microbiol Infect 1998;4:373-84.

35. Sanches I.S., Ramirez M., Troni H., et al. Evidence for the geographic spread of a methicillin-resistant Staphylococcus aureus clone between Portugal and Spain. J Clin Microbiol 1995;33:1243-6.

36. Souza M.A., Sanches I.S., Ferro M.L., et al. Intercontinental spread of a multidrug-resistant methicillin Staphylococcus aureus clone. J Clin Microbiol 1998;36:2590-6. 